\title{
Epigenetic priming of inflammatory response genes by high glucose in
}

\section{adipose progenitor cells}

Torunn Rønningen, Akshay Shah, Andrew H. Reiner, Philippe Collas* and Jan Øivind Moskaug*

Department of Molecular Medicine, Institute of Basic Medical Sciences, Faculty of Medicine, University of Oslo, and Norwegian Center for Stem Cell Research, Oslo University Hospital, Oslo, Norway

*Corresponding authors. Address: University of Oslo, Institute of Basic Medical Sciences, PO Box 1112 Blindern, 0317 Oslo, Norway. Fax: 47-22851058.

Email address: philc@medisin.uio.no (P.C.); j.o.moskaug@medisin.uio.no (J.Ø.M.)

RUNNING TITLE: Chromatin states of inflammation response genes 


\begin{abstract}
Cellular metabolism confers wide-spread epigenetic modifications required for regulation of transcriptional networks that determine cellular states. Mesenchymal stromal cells are responsive to metabolic cues including circulating glucose levels and modulate inflammatory responses. We show here that long term exposure of undifferentiated human adipose tissue stromal cells (ASCs) to high glucose upregulates a subset of inflammation response (IR) genes and alters their promoter histone methylation patterns in a manner consistent with transcriptional de-repression. Modeling of chromatin states from combinations of histone modifications in nearly 500 IR genes unveil three overarching chromatin configurations reflecting repressive, active, and potentially active states in promoter and enhancer elements. Accordingly, we show that adipogenic differentiation in high glucose predominantly upregulates IR genes. Our results indicate that elevated extracellular glucose levels sensitize in ASCs an IR gene expression program which is exacerbated during adipocyte differentiation. We propose that high glucose exposure conveys an epigenetic 'priming' of IR genes, favoring a transcriptional inflammatory response upon adipogenic stimulation. Chromatin alterations at IR genes by high glucose exposure may play a role in the etiology of metabolic diseases.
\end{abstract}

Keywords: Adipose stem cell, chromatin, gene expression, glucose, histone modification, inflammation 


\section{Introduction}

Chromatin remodeling plays an important role in establishing epigenetic patterns required for regulation of gene expression networks that determine cellular states. Cytosine methylation and histone post-translational modifications $\left(\mathrm{hPTMs}^{1}\right)$ sensitize the genome to changing environmental conditions including nutrient availability [1]. In addition, cellular metabolic pathways provide intermediates that serve as co-factors of histone modifying enzymes and can alter expression level of these enzymes [2]. Histone methyltransferases and deacetylases are metabolically responsive, altering patterns of histone $\mathrm{H} 3$ and $\mathrm{H} 4$ methylation or acetylation, respectively, on lysine (K) residues. Histone PTMs include trimethylation of $\mathrm{H} 3 \mathrm{~K} 4$ (H3K4me3), which marks transcription start sites (TSSs) of expressed and many non-expressed genes, H3K9 di- and trimethylation (H3K9me2/3) associated with inactive promoters, $\mathrm{H} 3 \mathrm{~K} 27 \mathrm{me} 3$, marking inactive promoters and enhancers, and forms of $\mathrm{H} 3$ and $\mathrm{H} 4$ acetylation on promoters and enhancers, often associated with transcriptional activity. Cellular metabolic state thus confers epigenetic modifications throughout the genome, including on promoters and enhancers, key regulators of gene expression.

Mesenchymal stromal cells (MSCs) are multipotent cells found in various tissues including adipose tissue [3], and are responsive to cellular metabolic states. MSCs modulate immune and inflammatory responses, in part by secreting cytokines acting as chemoattractants for monocytes and neutrophils $[4,5]$. Regulatory roles of adipose tissue-derived MSCs (ASCs) in inflammation [6] are likely influenced by metabolic cues such as high circulating blood glucose in individuals with insulin resistance [7]. Elevated extracellular glucose alters metabolic states and influences H3 methylation and acetylation [1]. Interestingly, promoters of inflammation response (IR) genes in cardiomyocytes [8] and endothelial cells [9] are modified through histone methylation in a glucose-dependent manner. These modifications may constitute an 'epigenetic memory' of an inflammatory predisposition [1], contributing to an increased IR after a metabolic insult. Little is known, however, on 
how nutrient availability and glucose levels in particular impact epigenetic modifications on genes involved in inflammatory processes in adipocyte progenitors and during adipogenic differentiation.

We show here that exposure of human primary ASCs to elevated glucose upregulates IR genes and alters $\mathrm{H} 3$ methylation in a manner suggesting transcriptional de-repression. Modeling of chromatin states from combinations of seven chromatin marks in 497 IR genes unveils chromatin configurations suggestive of potentially active states in promoter and enhancer elements. Accordingly, adipogenic induction in high glucose exacerbates transcriptional upregulation of IR genes. Our results suggest that elevated glucose epigenetically primes ASCs for upregulation of an inflammatory response upon adipogenic stimulation.

\section{Materials and methods}

Cells. ASCs were isolated from the stromal vascular fraction of human lipoaspirates after approval from the Norwegian Regional Ethics Committee (No. S06387a). Cells were cultured in DMEM/F12 (17.5 mM glucose; Gibco) containing 10\% fetal calf serum (FCS) [10]. For glucose treatment, ASCs were cultured in DMEM/F12 containing $5 \mathrm{mM}$ D-glucose (prepared by mixing DMEM. No. 11966025 and 21765029; Gibco), 10\% FCS and supplemented with D-glucose (SigmaAldrich) to 15 or $25 \mathrm{mM}$. Adipogenic differentiation was as described [11, 12]. Briefly, proliferating ASCs were plated confluent in DMEM/F12/10\% FCS. After $24 \mathrm{~h}$ medium was changed to DMEM/F12/10\% FCS containing 5, 15 or $25 \mathrm{mM}$ glucose. Adipogenesis was initiated after another $24 \mathrm{~h}$ and extended to 21 days.

Antibodies. Antibodies were anti-H3K4me3 (003-050, Diagenode), anti-H3K9me3 (056-050, Diagenode), anti-H3K27me3 (069-050, Diagenode), and anti- $\alpha$-tubulin (T5326, Sigma-Aldrich). An irrelevant IgG was used in control ChIPs (12-370, Millipore). 
Rønningen et al. 2015 BBRC, accepted

Western blotting. Cells were lysed in Laemmli buffer and proteins separated by SDS-PAGE and blotted on Immobilon-FL (Millipore). Membranes were blocked with Odyssey blocking buffer (Licor), incubated with antibodies and proteins visualized using IRDye-800-coupled secondary antibodies (Licor) on an Odyssey imaging system.

Reverse transcription (RT)-qPCR. Total RNA was extracted using GenElute ${ }^{\mathrm{TM}}$ Mammalian Total RNA Miniprep Kit (Sigma-Aldrich) and reverse-transcribed (iScript cDNA synthesis kit; Biorad). RT-qPCR was done in triplicates using the LightCycler $^{\circledR}$ 2.0 System (Roche) and indicated primers (Supplementary Table 1). mRNA levels were normalized to SF3A1 expression by the comparative CT method.

Expression microarrays. Total RNA was isolated and processed using Illumina TotalPrep RNA Amplification Kit (Ambion) for labeling and hybridization to Illumina HumanWG-6 v3 Expression BeadChips. Data were analyzed and quartile normalized using Illumina's GenomeStudio v2010.1, Gene Expression module v1.6.0. We called transcripts detected with $\mathrm{P} \geq 0.05$ as 'absent' and those detected with $\mathrm{P}<0.05$ as 'present'. For analysis of glucose effects, fold expression change cut-off for up- and down-regulated genes was set to 1.5. 'Inflammation response' GO terms were identified by searching GO database 2014-02-01 with AmiGO (http://amigo.geneontology.org/cgibin/amigo/go.cgi) for all terms related to GO:0006954. Analysis of enriched GO terms was done using DAVID 6.7 with $\mathrm{P}<0.001$. Microarray data are deposited under NCBI GEO GSE48774.

Chromatin immunoprecipitation. ChIP was done as described [13]. Briefly, $3 \times 10^{6}$ cells were cross-linked, lysed and sonicated. ChIP was done in duplicates using $2.5 \mu \mathrm{g}$ antibody. ChIP DNA was analyzed by qPCR on a MyiQ Real-time PCR Detection System as follows: $95^{\circ} \mathrm{C} 3 \mathrm{~min}$, and 40 cycles of $95^{\circ} \mathrm{C} 30 \mathrm{sec}, 60^{\circ} \mathrm{C} 30 \mathrm{sec}$, and $72^{\circ} \mathrm{C} 30 \mathrm{sec}$. Primers (Supplementary Table 2) were designed to amplify promoter regions based on HG18 genome assembly. 
Rønningen et al. 2015 BBRC, accepted

Analysis of chromatin states. ChIP-seq datasets of hPTMs used for chromatin state modeling were obtained from a previous study [14] using a similar source of ASCs and differentiation protocol. hPTM and CTCF enrichment data were converted into chromatin states in 200-base pair bins throughout the genome using ChromHMM [15]. Options were selected to learn a 15-state model using the Baum-Welch training algorithm as described [12]. To determine enrichment of gene regions (defined as gene $\pm 10 \mathrm{~kb}$ to accommodate promoter and (putative) enhancer elements, due to the nature of the hPTMs examined) in each chromatin state, we computed the ratio of coverage of a given state (chromatin state (cs)1-15) per base in the gene region normalized to coverage of that chromatin state in the whole genome. Heat maps of chromatin state enrichment per gene region were generated as a percentage of coverage of the gene region normalized by the length of the gene region. Genes with similar chromatin state profiles were clustered by hierarchical clustering using hclust and enrichment of states as variables [12]. The tree was generated using cutree. Scripts were written in Perl or R and plots generated using ggplot2 in R.

Statistics. Mean values from RT-qPCR and ChIP qPCR experiments were normalized to reference levels (fold change and fold enrichment values, respectively), and one sample t-test was used with $\mathrm{p}<0.05$ (IBM SPSS Statistics v19.0).

\section{Results and discussion}

Exposure of ASCs to high glucose alters histone methylation on promoters of inflammatory genes

To get a first appreciation of a glucose effect on expression of genes involved in inflammation responses, we examined transcripts of genes with a connection to hyperglycemia and diabetes, encoding cytokines and chemokines implicated in immune cell chemotaxis. We performed this analysis in human ASCs from three non-obese donors. Purified ASCs were cultured for 21 days (after thawing stocks) in 5, 15 or $25 \mathrm{mM}$ glucose. Genes examined included RARRES2 (chemerin), 


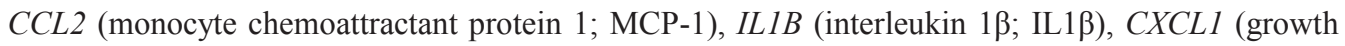
regulated oncogene $\alpha$; GRO $\alpha$ ), CXCL5 (chemokine [C-X-C motif] ligand 5) and IL8 (interleukin 8). RT-qPCR analysis shows that, while $15 \mathrm{mM}$ glucose confers a minor effect on expression of these genes compared to control cells cultured in $5 \mathrm{mM}$ glucose, $25 \mathrm{mM}$ glucose moderately enhances expression of all genes except CXCL5 (Supplementary Fig. 1). Thus, long term exposure of ASCs to elevated extracellular glucose upregulates a subset of IR genes. This is concordant with induced expression of pro-inflammatory factors in mouse 3T3-L1 adipocytes cultured in high glucose [16].

Increasing evidence links carbohydrate metabolism to changes in chromatin $[1,17]$. Thus we examined for each donor changes in hPTMs in the promoters of these genes (Supplementary Fig. 2A). ChIP-qPCR data show variable extents of reduction in H3K9me3 and H3K27me3 in 15 mM vs. $5 \mathrm{mM}$ glucose, that appear to be donor- and locus-dependent (Fig. 1A,B; Supplementary Fig. 2B). These reductions do not always coincide with changes in transcript levels. We find however, in 25 vs. $5 \mathrm{mM}$ glucose, an overall and more consistent reduction in $\mathrm{H} 3 \mathrm{~K} 9 \mathrm{me} 3$ and $\mathrm{H} 3 \mathrm{~K} 27 \mathrm{me}$, with no alteration in TSS marking by H3K4me3 (Fig. 1A,B; Supplementary Fig. 2B). These changes do not reflect alterations in global cellular levels of these hPTMs, as shown by Western blotting (Fig. 1C), but rather, the induction of a de-repressive mechanism at these sites. These hPTM changes generally correlate with an increase in transcript levels (Supplementary Fig. 1).

In vascular smooth muscle cells, reduced $\mathrm{H} 3 \mathrm{~K} 9 \mathrm{me} 3$ on the RELA promoter encoding the p65 subunit of inflammatory transcription factor $\mathrm{NF \kappa B}$ occurs in response to high glucose, an effect ascribed to histone methyltransferase SuV39h1 and lysine-specific demethylase LSD1 [8, 18, 19]. CXCL1, CXCL5, IL8 and IL1B promoter/enhancer regions contain predicted binding sites for NFKB whose binding may be modulated by methylation of H3K9 and K27. Little is known on the relationship between hyperglycemia and activity of EZH2, the H3K27 methyltransferase. Nonetheless, EZH2 activity is regulated by $O$-GlcNAcylation [20], supporting a link between 
glucose sensing and Polycomb-mediated gene repression via H3K27me3. Additionally, the H3K27me3 demethylase KDM6B promotes pro-inflammatory IL12 expression in bone marrow stem cells from diabetic patients [21], linking H3K27 methylation to inflammatory cellular states.

Chromatin state modeling unveils states of potentially active regulatory elements of inflammation response genes in ASCs

To investigate how elevated glucose might influence inflammatory gene transcription profiles in ASCs, we analyzed using cDNA microarrays the transcriptome of ASCs cultured under proliferation conditions (DMEM/F12; $17.5 \mathrm{mM}$ glucose). We focused our expression analysis on a set of 497 genes constituting the AmiGO gene ontology (GO) category 'Inflammatory response' (IR) (GO:0006954). Among these genes, 201 are commonly expressed in ASCs from all three donors (Fig. 2A; see Supplementary Table 3 for gene lists). Four of the six genes in our initial RT-qPCR analyses of hyperglycemia and diabetes relevant genes (Supplementary Fig. 1A: RARRES2, CCL2, $I L 8$ and $I L 1 B$ ) were found expressed in the microarray analysis. Supporting these findings, our mRNA-sequencing data from a separate ASC culture [12] in $17.5 \mathrm{mM}$ glucose confirm the higher expression level of the 201 IR genes identified above relative to all genes in the genome (Fig. 2B). Thus under standard in vitro culture conditions, ASCs express genes encoding inflammatory modulators; nonetheless expectedly, many IR genes are also not expressed.

To evaluate the molecular basis for differential expression of IR genes, we determined the 'chromatin states' of the 497 IR genes, i.e. combinations of chromatin modifications as they occur at and around these genes in ASCs. To this end, we used ChromHMM [15], a Hidden Markov Modeling algorithm we recently applied to identify chromatin states in ASCs, from recurrent combinations of 7 chromatin marks, including 6 hPTMs (H3K4me1, H3K4me2, H3K4me3, H3K27me3, H3K27ac, H3K36me3) and CCCTC-binding factor (CTCF) binding [12]. These marks 
have been previously mapped by ChIP-sequencing (ChIP-seq) in human ASCs [14] using a similar culture system and modeled by us into a 15 -chromatin state model, that is, 15 distinct combinations of these marks [12].

Composition of the 15 chromatin states modeled is shown in Figure 3A. Using this model, we determined the relative enrichment level of each state at and around each of the 497 IR genes. These 497 gene regions included the actual genes \pm 10 kilobases $(\mathrm{kb})$ upstream and downstream as a proxy to accommodate promoters and (putative) enhancer elements [12]. Chromatin state enrichment in a given gene region was calculated as the ratio of abundance of each state in the region relative to the abundance of the same state in the whole genome (Fig. 3B). Thus a ratio of 1 reflects the detection of a given chromatin state but no enrichment in the gene region. Results show a predominant enrichment of chromatin state (cs) 5, annotated as 'active enhancer in gene body' (Fig. 3A), composed of $\mathrm{H} 3 \mathrm{~K} 36 \mathrm{me} 3, \mathrm{H} 3 \mathrm{~K} 4 \mathrm{me} 1 / \mathrm{me} 2$ and $\mathrm{H} 3 \mathrm{~K} 27 \mathrm{ac}$ (Fig. 3B). Enrichment in cs5 is consistent with a subset of IR genes being expressed. Accordingly, we note a predominance of chromatin states characteristic of active genes (cs6) and active promoters and enhancers (cs10-14; Fig. 3B). We note an incidence of 'blank' cs7 (devoid of any of the marks examined) and cs 8 which marks inactive promoters (H3K27me3), in line with a significant proportion of the 497 IR genes not being expressed. Interestingly however, we also detect chromatin states suggestive of potential for gene expression, such as enhancer marking by $\mathrm{H} 3 \mathrm{~K} 4 \mathrm{me} 1(\mathrm{cs} 3, \mathrm{cs} 4)$ and a transcriptionally 'poised' promoter state marked by $\mathrm{H} 3 \mathrm{~K} 4 \mathrm{me} 2 / 3$ and $\mathrm{H} 3 \mathrm{~K} 27 \mathrm{me} 3$ (cs9). This suggests that a subset of IR genes held in a repressed state in ASCs harbors a potential for transcriptional activation. This raises the possibility that this poised potential for gene expression constitutes an epigenetic 'priming' of IR genes.

To further characterize chromatin states of the non-expressed IR genes in ASCs, we next grouped the 497 IR genes into a cluster consisting of the 201 genes expressed in all three donors 
examined above (we call this cluster the '201-cluster'; see Fig. 2A) and into a cluster containing the remaining 296 genes ('296-cluster'). We then computed within each cluster the proportions of each gene region covered by each chromatin state, and generated a normalized chromatin state heat map for each gene (Fig. 3C; Supplementary Table 4). Consistent with its expressed status, the 201-cluster shows a high incidence of cs6 (H3K36me3 on gene bodies), cs4 (H3K4me1), and combinations of H3K4me2, H3K4me3 and H3K27ac on promoters (cs2, cs10, cs13; Fig. 3C, left). In contrast, in line with the overall repressed status of the 296-cluster, active promoter and enhancer states (cs1-3, cs1014) are of low abundance (Fig. 3C, right). However, enhancer (H3K4me1; cs3, cs4) and promoter (H3K4me2 or me3; cs10) marking is prominent, along with H3K27me3 (cs8; Fig. 3C, right).

To qualify these observations in an unbiased manner, we performed a hierarchical clustering of genes in the 296-cluster based on chromatin state abundance in the gene regions. Due to its overarching abundance in this cluster (see Fig. 3C, right), cs7 ('blank state') was removed from this clustering. This analysis highlights 3 sub-clusters (clusters 1-3) containing $88 \%$ of the whole 296 gene set (Fig. 3D; Supplementary Fig. 3; Supplementary Table 5). Most genes are clustered based on the marking of enhancers (cluster 1: cs3 and cs4; Fig. 3D); these genes are repressed, as shown by overall depletion of cs6 (H3K36me3) and enrichment in $\operatorname{cs} 8(\mathrm{H} 3 \mathrm{~K} 27 \mathrm{me} 3)$. Cluster 1 is also characterized by cs10, reflecting the marking of TSSs by di- or trimethylated H3K4. Cluster 2 is enriched in similar states (cs3, 4, 10) and cs6 (H3K36me3 marking), consistent with a subset of these genes being expressed. We further note a smaller group of 20 genes clustered based on repressive promoter marking by $\mathrm{H} 3 \mathrm{~K} 27 \mathrm{me} 3$ (cluster 3: cs8; Fig. 3D), in line with the overall transcriptionally inactive status of the 296-cluster.

We conclude that in ASCs cultured in $17.5 \mathrm{mM}$ glucose, a large set of IR genes is not expressed but harbors promoters and enhancers marked by hPTMs suggestive of potential for transcriptional activation; thus, these genes lie in chromatin environment permissive for expression. 
This is consistent with the immuno-regulatory properties of cells in adipose tissue [22] and with their response to metabolic changes, such as development of insulin resistance [23]. This condition results in hyperglycemia and cellular exposure to excess glucose, leading to an inflammatory response.

High extracellular glucose upregulates IR genes in undifferentiated ASCs, an effect exacerbated by adipogenic differentiation

The transcriptionally permissive state of the IR genes identified above suggests that these genes may be responsive to stimulation by elevated glucose. Testing this hypothesis, we monitored the transcriptome of undifferentiated ASCs cultured in 5 or $25 \mathrm{mM}$ glucose for 21 days. We strikingly find that the top 7 enriched GO terms for upregulated genes ( $>1.5$-fold change) in $25 \mathrm{mM}$ glucose, compared to $5 \mathrm{mM}$ glucose, are associated with signaling and inflammatory responses (Fig. 4A; Supplementary Table 6). Therefore, long term exposure of undifferentiated ASCs to high glucose favors upregulation of IR genes. Downregulated genes relate to mitotic regulation (Supplementary Fig. 4A; Supplementary Table 6), reflecting a negative impact of prolonged culture in high glucose on cell cycle progression.

We next asked whether adipogenic stimulation in high glucose would exacerbate IR gene upregulation. This system would model a connection between adipose tissue expansion and low grade adipocyte inflammation, which has been suggested earlier [24]. We examined using microarrays the transcriptome of ASCs differentiated into adipocytes for 21 days in adipogenic medium containing 5 or $25 \mathrm{mM}$ glucose. As a control, ASCs were maintained under proliferation conditions in 5 or $25 \mathrm{mM}$ glucose. ASCs differentiated efficiently regardless of glucose concentration, as judged by Oil Red-O staining of lipid droplets and by RT-qPCR analysis of adipogenic transcripts (Supplementary Fig. 5). Strikingly however, among genes significantly upregulated by adipogenic stimulation in $25 \mathrm{mM}$ relative to $5 \mathrm{mM}$ glucose, we find that the top 10 
enriched GO terms involve IR and signaling functions (Fig. 4B; Supplementary Table 7). In addition, we find more upregulated genes enriched in the 'Inflammatory response' GO category (GO:0006954) in $25 \mathrm{mM}$ glucose adipocytes than in non-differentiated $25 \mathrm{mM}$ glucose ASCs (Fig. 4C; Supplementary Table 7). Similar data were obtained in $15 \mathrm{mM}$ ASCs and adipocytes (Supplementary Fig. 6, Supplementary Tables 6 and 7). Therefore, adipogenic stimulation in high glucose exacerbates inflammation-related transcription responses. Our findings agree with a view of high glucose enhancing an epigenetic predisposition of ASCs to elicit IR gene activation upon adipogenic stimulation.

Interestingly, we note in undifferentiated ASCs and adipocytes exposed to $25 \mathrm{mM}$ glucose, upregulation of IL6 (Fig. 4C; Supplementary Fig. 6E). IL6 conveys the immunomodulatory effect of MSCs [25] and is implicated in inflammation disease states including diabetes mellitus [26]. Additional genes upregulated in high glucose ASCs (AOX1, HMOX1, NFKBIZ and FOS) and adipocytes (NOX4, CYBA and FOS) are involved in oxidative stress, a major driver of inflammation (Fig. 4C).

Our observations support a notion that inflammation status of adipose tissue is exacerbated by circulating high blood glucose and that IR genes are metabolically regulated at least partly by epigenetic mechanisms. We propose a model (Fig. 4D) where glucose-dependent reduction or loss of repressive $\mathrm{H} 3$ modifications on IR gene regulatory elements may be involved in epigenetic 'priming' in ASCs, conferring IR genes with a transcriptionally permissive state. This may lead to exacerbated upregulation of a transcriptional inflammatory gene network after stimulus such as adipogenic induction in high glucose (Fig. 4D). Our results are consistent with a view of cellular environment providing contextual determinants of epigenetic modifications that may prime or drive transcriptional networks controlling inflammatory responses in mesenchymal progenitor cells. 
${ }^{1}$ Abbreviations: ASC, adipose tissue-derived MSC; ChIP, chromatin immunoprecipitation; GO, gene ontology; IR, inflammation response; MSC, mesenchymal stromal cell; TSS, transcription start site

\section{Acknowledgments}

Work was supported by the Research Council of Norway, The Norwegian Center for Stem Cell Research and The University of Oslo.

\section{Data linking}

Expression data are deposited under NCBI GEO GSE48774.

\section{References}

[1] S.T. Keating, A. El-Osta, Chromatin modifications associated with diabetes, J Cardiovasc Transl Res, 5 (2012) 399-412.

[2] S.T. Keating, A. El-Osta, Epigenetics and metabolism, Circ Res, 116 (2015) 715-736.

[3] L. da Silva Meirelles, P.C. Chagastelles, N.B. Nardi, Mesenchymal stem cells reside in virtually all post-natal organs and tissues, J Cell Sci, 119 (2006) 2204-2213.

[4] A.J. Nauta, W.E. Fibbe, Immunomodulatory properties of mesenchymal stromal cells, Blood, 110 (2007) 3499-3506.

[5] H.K. Salem, C. Thiemermann, Mesenchymal stromal cells: current understanding and clinical status, Stem Cells, 28 (2010) 585-596.

[6] D.J. Prockop, J.Y. Oh, Mesenchymal stem/stromal cells (MSCs): role as guardians of inflammation, Mol Ther, 20 (2012) 14-20.

[7] B. Balhara, A. Burkart, V. Topcu, Y.K. Lee, C. Cowan, C.R. Kahn, M.E. Patti, Severe insulin resistance alters metabolism in mesenchymal progenitor cells, Endocrinol, 156 (2015) 2039-2048.

[8] L.M. Villeneuve, M.A. Reddy, L.L. Lanting, M. Wang, L. Meng, R. Natarajan, Epigenetic histone H3 lysine 9 methylation in metabolic memory and inflammatory phenotype of vascular smooth muscle cells in diabetes, Proc Nat Acad Sci USA, 105 (2008) 9047-9052.

[9] L. Pirola, A. Balcerczyk, R.W. Tothill, I. Haviv, A. Kaspi, S. Lunke, M. Ziemann, T. Karagiannis, S. Tonna, A. Kowalczyk, B. Beresford-Smith, G. Macintyre, M. Kelong, Z. Hongyu, J. Zhu, A. ElOsta, Genome-wide analysis distinguishes hyperglycemia regulated epigenetic signatures of primary vascular cells, Genome Res, 21 (2011) 1601-1615.

[10] A.C. Boquest, A. Shahdadfar, K. Fronsdal, O. Sigurjonsson, S.H. Tunheim, P. Collas, J.E. Brinchmann, Isolation and transcription profiling of purified uncultured human stromal stem cells: alteration of gene expression after in vitro cell culture, Mol Biol Cell, 16 (2005) 1131-1141. 
[11] J.L. Boulland, M. Mastrangelopoulou, A.C. Boquest, R. Jakobsen, A. Noer, J.C. Glover, P. Collas, Epigenetic regulation of nestin expression during neurogenic differentiation of adipose tissue stem cells, Stem Cells Dev, 22 (2013) 1042-1052.

[12] A. Shah, A. Oldenburg, P. Collas, A hyper-dynamic nature of bivalent promoter states underlies coordinated developmental gene expression modules, BMC Genomics, 15 (2014) 1186.

[13] J.A. Dahl, P. Collas, A quick and quantitative chromatin immunoprecipitation assay for small cell samples, Front Bioscience, 12 (2007) 4925-4931.

[14] T.S. Mikkelsen, Z. Xu, X. Zhang, L. Wang, J.M. Gimble, E.S. Lander, E.D. Rosen, Comparative epigenomic analysis of murine and human adipogenesis, Cell, 143 (2010) 156-169.

[15] J. Ernst, M. Kellis, ChromHMM: automating chromatin-state discovery and characterization, Nat Methods, 9 (2012) 215-216.

[16] Y. Lin, A.H. Berg, P. Iyengar, T.K. Lam, A. Giacca, T.P. Combs, M.W. Rajala, X. Du, B. Rollman, W. Li, M. Hawkins, N. Barzilai, C.J. Rhodes, I.G. Fantus, M. Brownlee, P.E. Scherer, The hyperglycemia-induced inflammatory response in adipocytes: the role of reactive oxygen species, $\mathrm{J}$ Biol Chem, 280 (2005) 4617-4626.

[17] C. Lu, C.B. Thompson, Metabolic regulation of epigenetics, Cell Metab, 16 (2012) 9-17.

[18] D. Brasacchio, J. Okabe, C. Tikellis, A. Balcerczyk, P. George, E.K. Baker, A.C. Calkin, M. Brownlee, M.E. Cooper, A. El-Osta, Hyperglycemia induces a dynamic cooperativity of histone methylase and demethylase enzymes associated with gene-activating epigenetic marks that coexist on the lysine tail, Diabetes, 58 (2009) 1229-1236.

[19] A. El-Osta, D. Brasacchio, D. Yao, A. Pocai, P.L. Jones, R.G. Roeder, M.E. Cooper, M. Brownlee, Transient high glucose causes persistent epigenetic changes and altered gene expression during subsequent normoglycemia, J Exp Med, 205 (2008) 2409-2417.

[20] C.S. Chu, P.W. Lo, Y.H. Yeh, P.H. Hsu, S.H. Peng, Y.C. Teng, M.L. Kang, C.H. Wong, L.J. Juan, O-GlcNAcylation regulates EZH2 protein stability and function, Proc Nat Acad Sci USA, 111 (2014) 1355-1360.

[21] K.A. Gallagher, A. Joshi, W.F. Carson, M. Schaller, R. Allen, S. Mukerjee, N. Kittan, E.L. Feldman, P.K. Henke, C. Hogaboam, C.F. Burant, S.L. Kunkel, Epigenetic changes in bone marrow progenitor cells influence the inflammatory phenotype and alter wound healing in type 2 diabetes, Diabetes, 64 (2015) 1420-1430.

[22] J.R. Brestoff, D. Artis, Immune regulation of metabolic homeostasis in health and disease, Cell, 161 (2015) 146-160.

[23] J.M. Perkins, N.G. Joy, D.B. Tate, S.N. Davis, Acute effects of hyperinsulinemia and hyperglycemia on vascular inflammatory biomarkers and endothelial function in overweight and obese humans, Am J Physiol Endocrinol Metab, (2015) ajpendo 0006402015.

[24] I. Wernstedt Asterholm, C. Tao, T.S. Morley, Q.A. Wang, F. Delgado-Lopez, Z.V. Wang, P.E. Scherer, Adipocyte inflammation is essential for healthy adipose tissue expansion and remodeling, Cell Metab, 20 (2014) 103-118.

[25] E.J. Bassi, D.C. de Almeida, P.M. Moraes-Vieira, N.O. Camara, Exploring the role of soluble factors associated with immune regulatory properties of mesenchymal stem cells, Stem Cell Rev, 8 (2012) 329-342. 
Rønningen et al. 2015 BBRC, accepted

[26] D. Qu, J. Liu, C.W. Lau, Y. Huang, IL-6 in diabetes and cardiovascular complications, Brit J Pharmacol, 171 (2014) 3595-3603. 


\section{Figures}

A

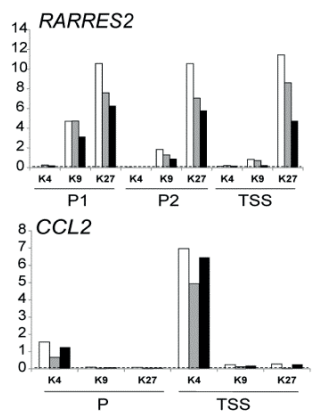

2.5 IL 1B

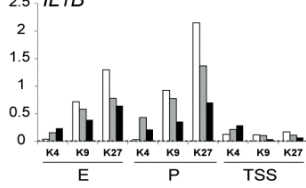

2.5 $\mathrm{CXCL} 1$
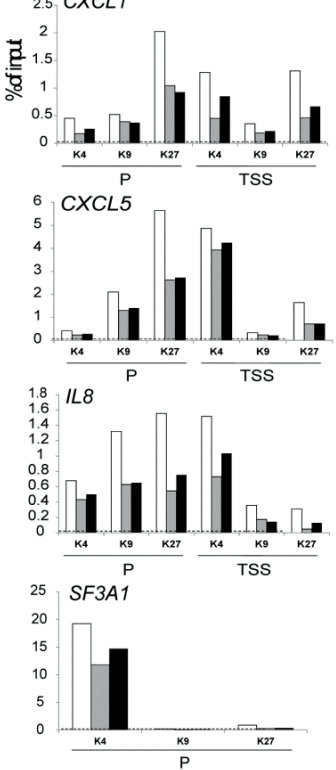

B
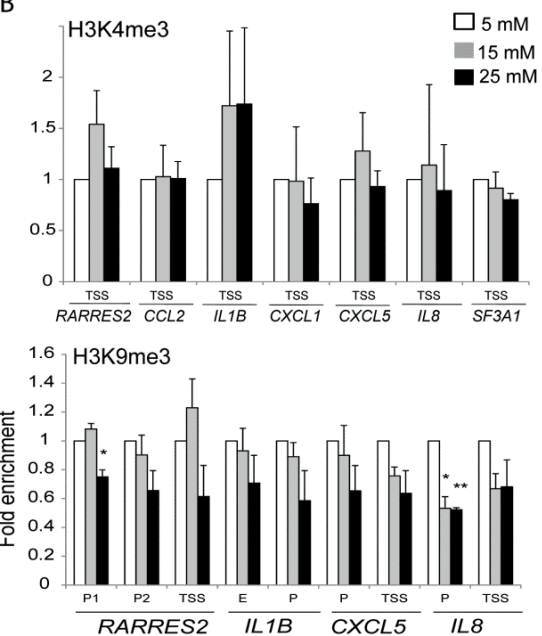

1.4 $\mathrm{H}$ H3K27me3

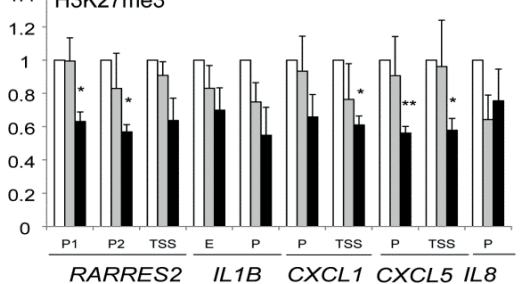

C

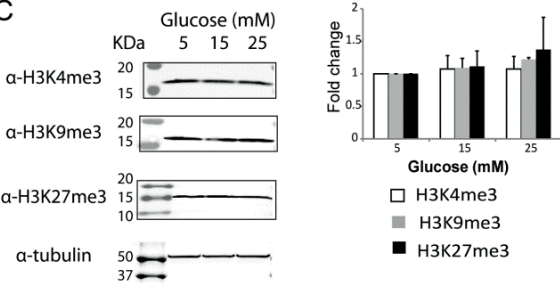

Fig. 1. High glucose elicits changes in $\mathrm{H} 3 \mathrm{~K} 4 \mathrm{me} 3, \mathrm{H} 3 \mathrm{~K} 9 \mathrm{me} 3$ and $\mathrm{H} 3 \mathrm{~K} 27 \mathrm{me} 3$ on inflammation response (IR) gene regulatory elements. (A) ChIP-qPCR of H3K4me3, H3K9me3 and H3K27me3 on indicated promoters in ASCs cultured in 5,15 or $25 \mathrm{mM}$ glucose for 21 days (legend in B). Data are shown as percent of input for one donor (see Supplementary Fig. 2B for data for the other donors). Mean enrichment levels in control IgG ChIPs are shown with dashed lines. (B) Combined 
ChIP results from three donors, expressed as fold enrichment relative to cells in $5 \mathrm{mM}$ glucose. Results are shown for regions where detection was higher than background (IgG) for all donors (mean \pm SEM from 3 experiments; $* \mathrm{P}<0.05$; $* * \mathrm{P}<0.01$, one sample t-test). (C) Western blots of $\mathrm{H} 3 \mathrm{~K} 4 \mathrm{me} 3, \mathrm{H} 3 \mathrm{~K} 9 \mathrm{me} 3, \mathrm{H} 3 \mathrm{~K} 27 \mathrm{me} 3$ and $\alpha$-tubulin as loading control. Graph shows quantifications relative to tubulin; mean $\pm \mathrm{SD}$ of 3 experiments. 
A

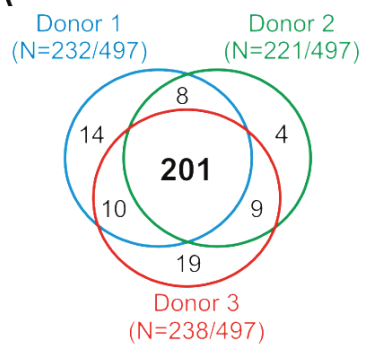

B

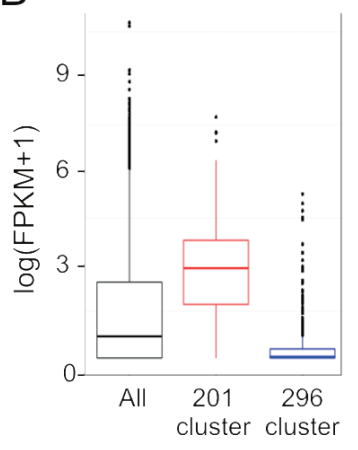

Fig. 2. A large subset of IR genes is expressed in ASCs. (A) Expression profiles of IR genes in ASCs from three donors cultured in $17.5 \mathrm{mM}$ glucose medium. IR genes $(\mathrm{n}=497)$ were identified from AmiGO, GO term GO:0006954. Transcribed genes were identified for each donor with $\mathrm{P}<0.05$. Venn diagram shows numbers of expressed IR transcripts. (B) Median gene expression levels (FPKM from RNA-seq data) of the 201- and 296-gene clusters and of all RefSeq genes. 
A

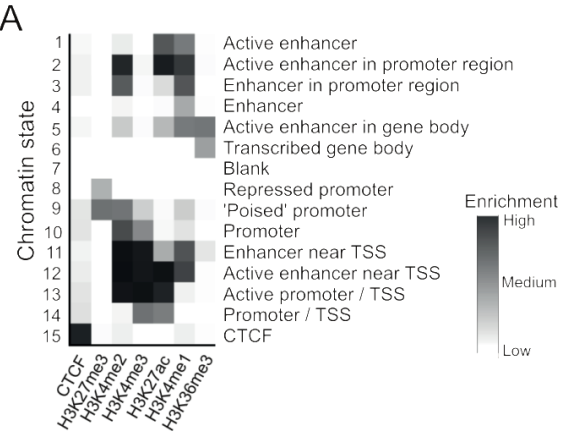

$\mathrm{B}$

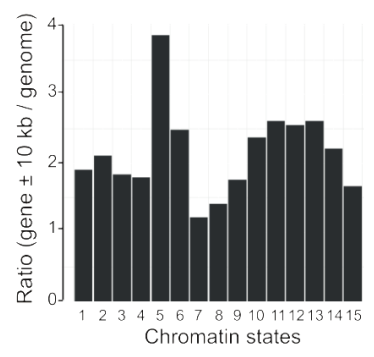

C

497 Inflammatory response genes
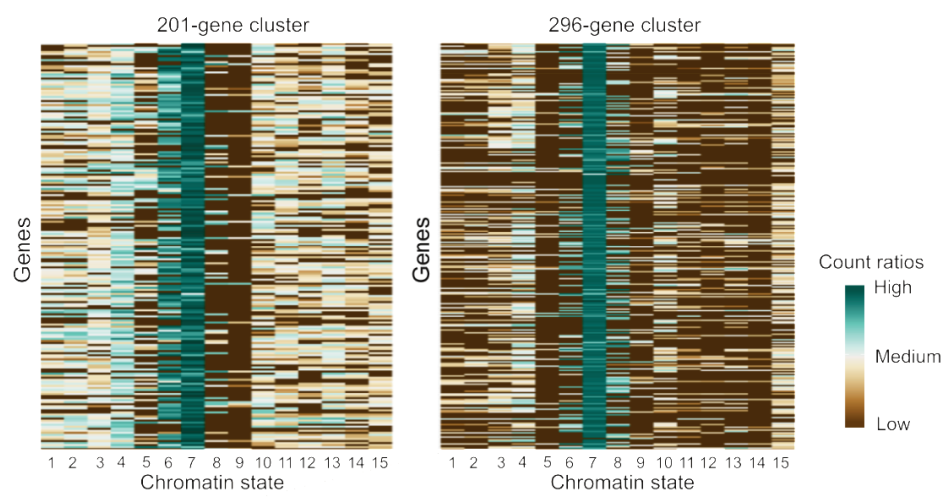

$\mathrm{D}$

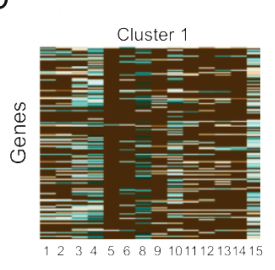

296-gene cluster

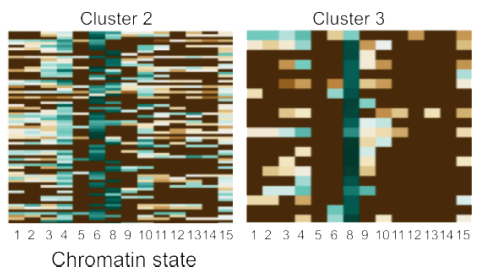

Fig. 3. Chromatin state analyses of IR genes in ASCs. (A) A 15 chromatin state model identified from combinations of $6 \mathrm{hPTMs}$ and CTCF binding. We used the same chromatin states as we previously reported [12] and functionally annotated each state (right). Relative enrichment level of chromatin marks constituting each chromatin state is shown as a heat map. (B) Enrichment of individual chromatin states in gene regions. (C) Chromatin state maps for each gene in the 201- and 296-clusters. A gene is defined as 'gene $\pm 10 \mathrm{~kb}$ '. Chromatin state enrichment is normalized to gene region length. (D) Chromatin state maps of genes in the 3 sub-clusters of the 296-cluster highlighted in Supplementary Fig. 3. These clusters contain $88 \%$ of genes in the 296-cluster. Scale shown in (C) is also valid for $(\mathrm{D})$. 


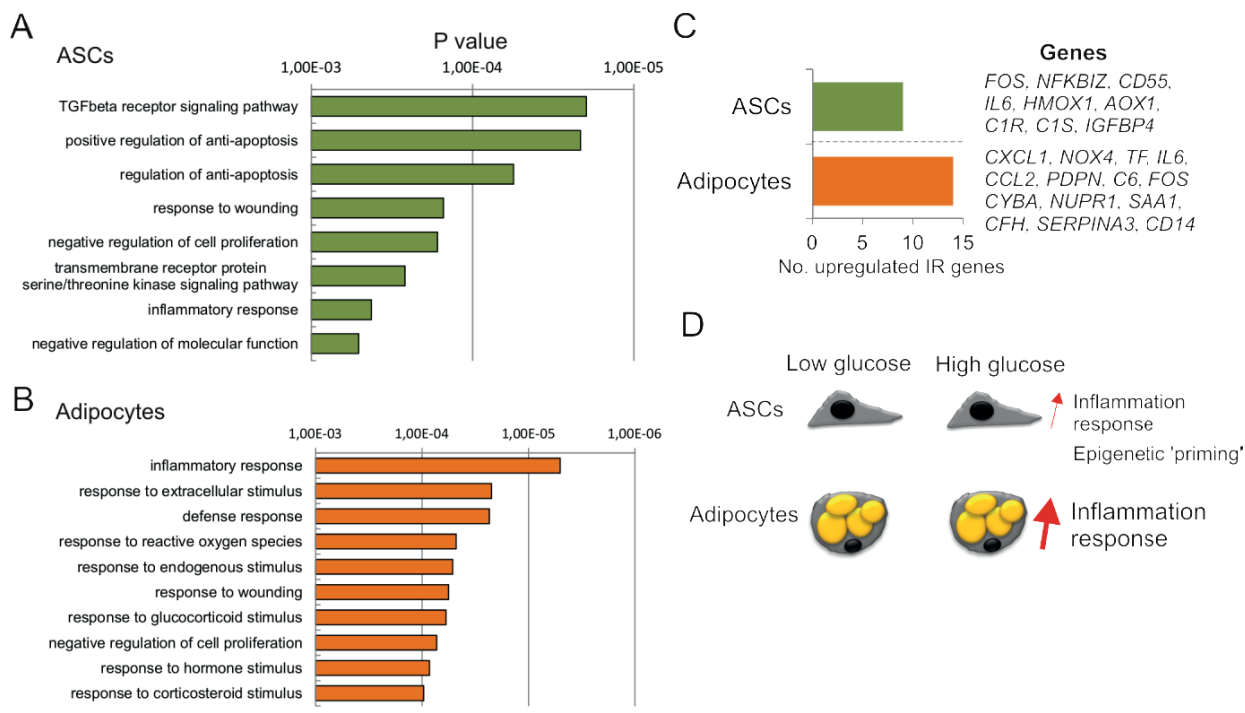

(Upregulated genes)

Fig. 4. GO term analysis of genes upregulated in 25 vs. $5 \mathrm{mM}$ glucose in ASCs and adipocytes reveal enrichment of IR genes. (A,B) Enriched GO terms for genes upregulated in ASCs (A) and adipocytes (B) after culture in $25 \mathrm{mM}$ relative to $5 \mathrm{mM}$ glucose; top $10 \mathrm{GO}$ terms; $\mathrm{P}<0.001$. (C) Numbers and names of upregulated IR genes in ASCs and adipocytes. (D) Model: high glucose induces epigenetic priming of IR genes in undifferentiated ASCs, leading to exacerbated IR gene upregulation after adipogenic differentiation. 
Rønningen et al.

Supplemental Material

Epigenetic priming of inflammatory response genes by high glucose in adipose progenitor cells

Torunn Rønningen, Akshay Shah, Andrew H. Reiner, Philippe Collas* and Jan Øivind Moskaug*

Department of Molecular Medicine, Institute of Basic Medical Sciences, Faculty of Medicine,

University of Oslo, 0317 Oslo, Norway

Supplementary Information 


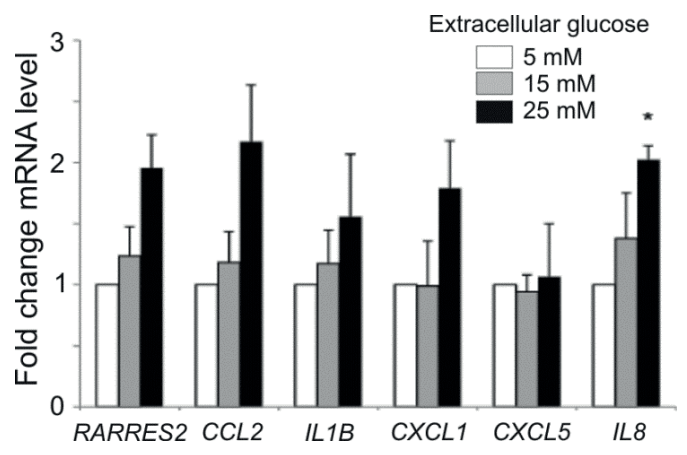

Supplementary Fig. 1. High glucose upregulates inflammation-associated transcripts in ASCs. RT-qPCR analysis of indicated inflammatory genes in ASCs from three different donors. Data are expressed as fold change expression in cells exposed to $15 \mathrm{mM}$ or $25 \mathrm{mM}$ glucose relative to a control $5 \mathrm{mM}$ glucose concentration (mean \pm SEM from 3 experiments; ${ }^{*} \mathrm{P}<0.05$; one sample $\mathrm{t}$ test). 
Rønningen et al.

Supplemental Material

A

RARRES2

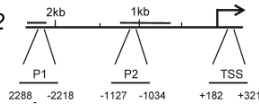

CCL2
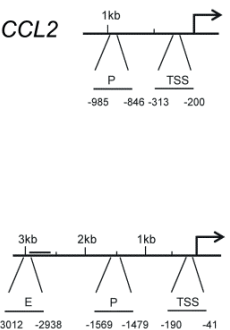

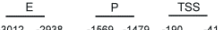
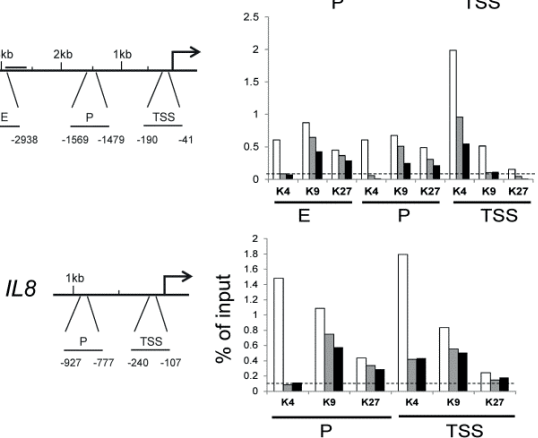

CXCL1
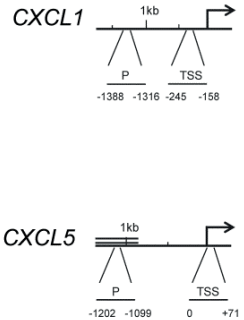

SF3A1

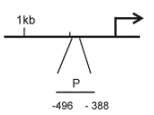

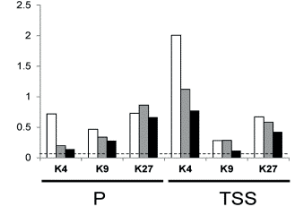

B

Donor 2
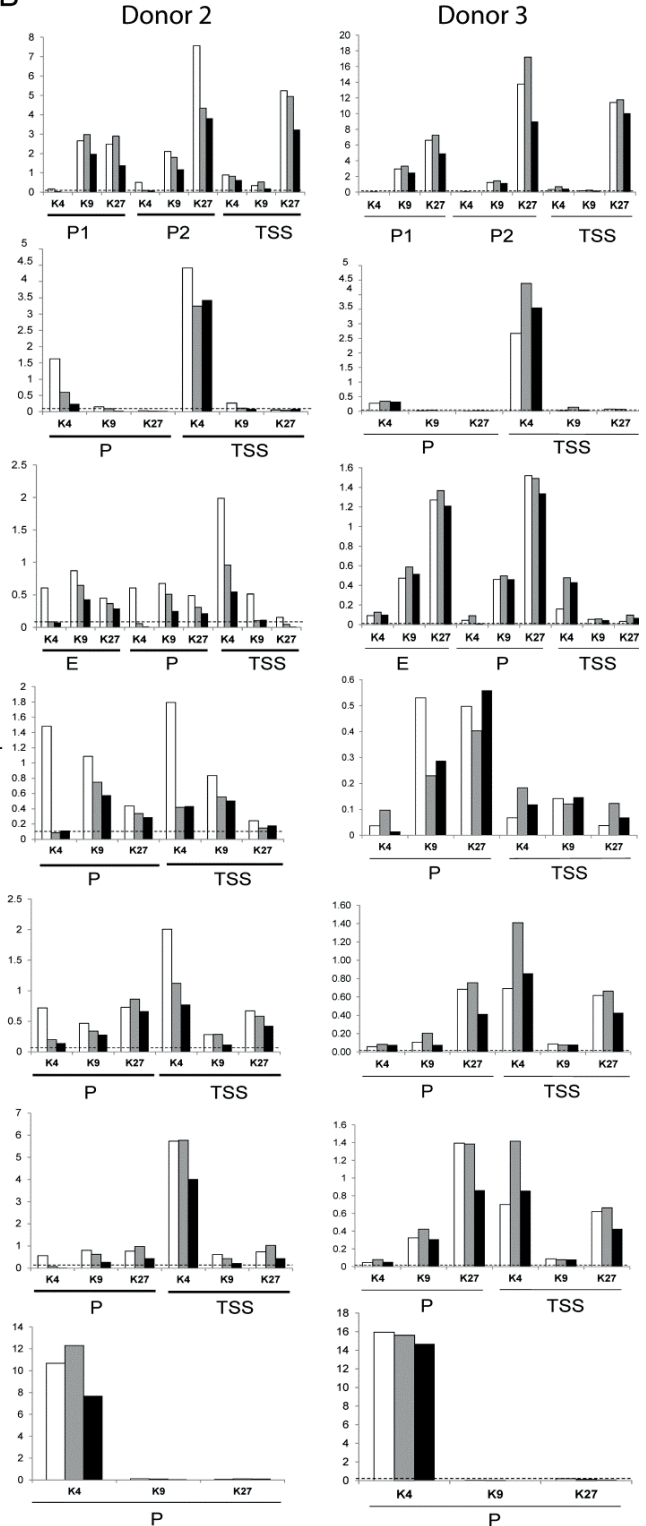

Supplementary Fig. 2. ChIP-qPCR analysis of H3K4me3, H4K9me3 and H3K27me3 in promoter regions of indicated genes for two ASC donors. (A) Positioning of PCR amplicons (numbers 
Rønningen et al.

Supplemental Material

indicate nucleotides relative to TSS [+1]). Regions found to be enriched in H3K4me3, H3K9me3 or H3K27me3 in a previously published ChIP-chip dataset from ASCs (Sørensen et al. 2010 Mol Biol Cell 21, 2066-2077) are indicated. Arrows mark TSSs and direction of transcription. (B) ChIPqPCR results for donors 2 and 3 (duplicate ChIPs each analyzed by duplicate qPCR). Horizontal dashed lines indicate average background enrichment level measured in control ChIPs using an irrelevant IgG. 
Rønningen et al.

Supplemental Material

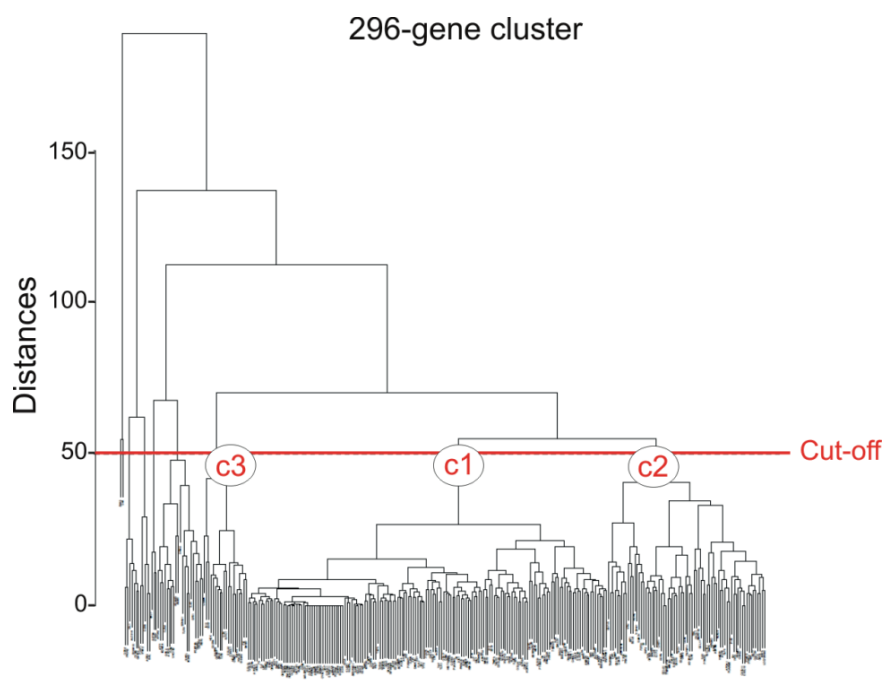

Supplementary Fig. 3. Hierarchical clustering of genes of the '296 cluster' based on chromatin state composition of genes in the cluster. The tree highlights three sub-clusters (c1, c2, c3) containing $88 \%$ of all genes in the 296 cluster. Chromatin state heat maps of clusters 1-3 are shown in Figure 2D. 
Rønningen et al.

Supplemental Material

A AsCs

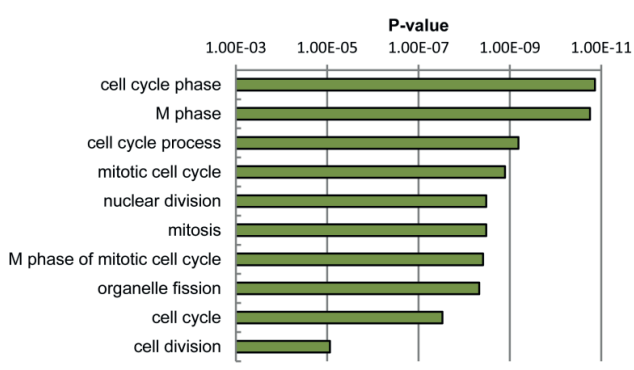

B Adipocytes

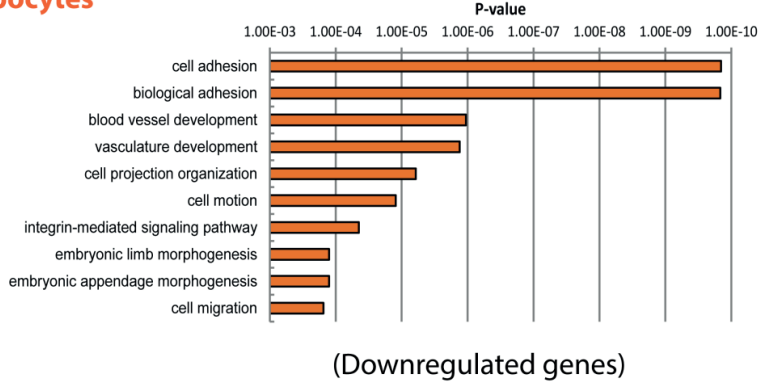

Supplementary Fig. 4. GO term enrichment for downregulated genes in (A) ASCs and (B) adipocytes after culture or differentiation, respectively, in $25 \mathrm{mM}$ glucose, relative to $5 \mathrm{mM}$ glucose. Fold change expression $>1.5 ; \mathrm{p}<0.001$; top 10 enriched GO terms are shown (DAVID 6.7). 

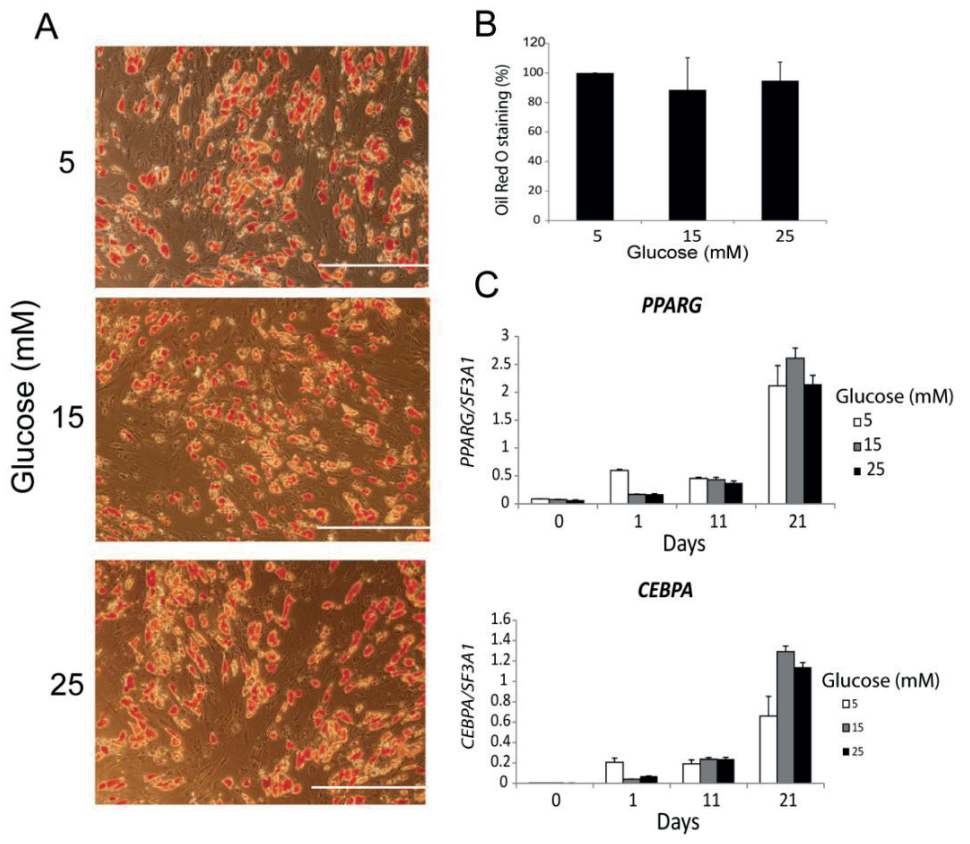

Supplementary Fig. 5. High glucose does not affect adipogenic differentiation capacity. ASCs were differentiated into adipocytes in medium containing 5, 15 or $25 \mathrm{mM}$ for 21 days. (A) Oil RedO staining of intracellular lipid droplets. Bars, $500 \mu \mathrm{m}$. (B) Quantification of Oil Red-O staining, related to total protein level (mean $\pm \mathrm{SD}$ from 3 independent experiments). (C) RT-qPCR analysis of expression of PPARG and CEBPA on day 1, 11 and 21 of adipogenic differentiation; data are expressed relative to $S F 3 A 1$ (mean \pm SD from 3 experiments). 
A

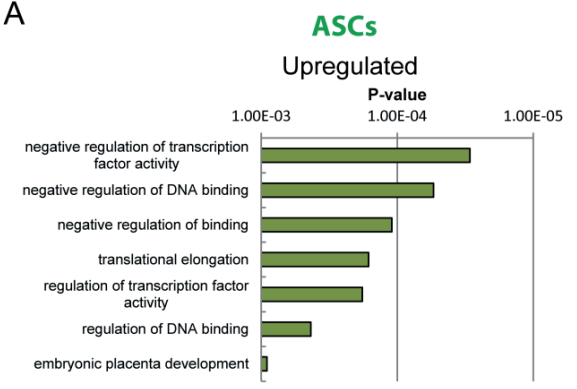

B

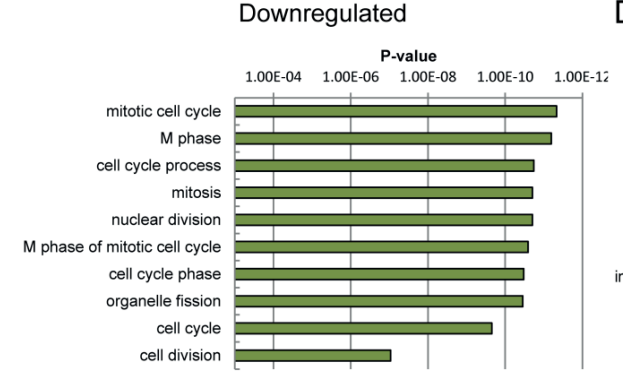

C

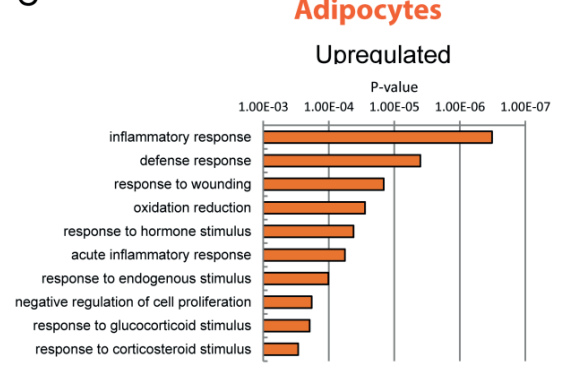

D

\section{Downregulated}

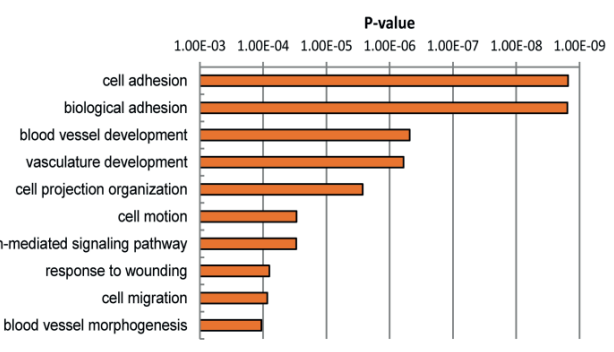

E

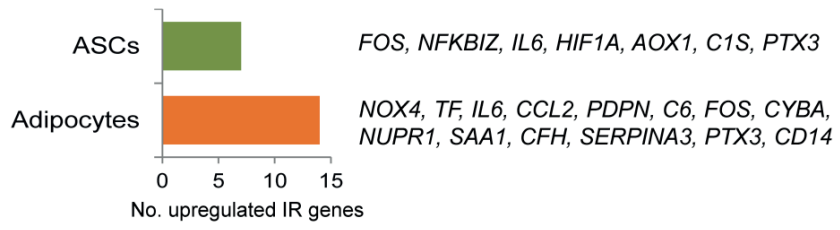

Supplementary Fig. 6. GO term enrichment for genes differentially expressed in ASCs and adipocytes cultured or differentiated, respectively, in $15 \mathrm{mM}$ relative to $5 \mathrm{mM}$ glucose. (A,B) Enriched GO terms for (A) upregulated and (B) downregulated genes in ASCs. (C,D) Enriched GO terms for (C) upregulated and (D) downregulated genes in 21-day adipocytes. (E) Number and name of upregulated IR genes in ASCs and adipocytes. Genes are found in the GO category "Inflammation response" (DAVID 6.7); for ASCs, this category is not significantly enriched $(\mathrm{p}=0.048)$. For adipocytes, this GO term is the most significant among upregulated genes (see panel C). Fold change expression $>1.5 ; \mathrm{p}<0.001$; top 10 enriched GO terms are shown (DAVID 6.7) 
Rønningen et al.

Supplemental Material

Supplementary Table 1. RT-qPCR primer used in this study

\begin{tabular}{|c|c|c|}
\hline Gene & Sequence & Product size \\
\hline RARRES2 & $\begin{array}{l}\text { F:AAACCCGAGTGCAAAGTCAG } \\
\text { R:CCGCAGAACTTGGGTCTCTA }\end{array}$ & 126 \\
\hline CCL2 & $\begin{array}{l}\text { F: TCTGTGCCTGCTGCTCATAG } \\
\text { R:AGCTTCTTTGGGACACTTGC }\end{array}$ & 169 \\
\hline IL1B & $\begin{array}{l}\text { F: CTCCAGGGACAGGATATGGA } \\
\text { R:TCATCTTTCAACACGCAGGA }\end{array}$ & 137 \\
\hline CXCL1 & $\begin{array}{l}\text { F: ATTCACCCCAAGAACATCCA } \\
\text { R:TAACTATGGGGGATGCAGGA }\end{array}$ & 127 \\
\hline CXCL5 & $\begin{array}{l}\text { F:AATTTTGGACGGTGGAAACA } \\
\text { R:TGTCTTCCCTGGGTTCAGAG }\end{array}$ & 114 \\
\hline IL8 & $\begin{array}{l}\text { F:CTGCGCCAACACAGAAATTA } \\
\text { R:TGAATTCTCAGCCCTCTTCAA }\end{array}$ & 118 \\
\hline SF3A1 & $\begin{array}{l}\text { F: CAAAATGCAGGAACACATGC } \\
\text { R: AGACCTGGTGCGTACACCTC }\end{array}$ & 111 \\
\hline PPARG2 & $\begin{array}{l}\text { F: TTCCTGGTTTTGTAAATCTTGACT } \\
\text { R: TTGTGTAAATGATCTCGTGGACT }\end{array}$ & 78 \\
\hline CEBPA & $\begin{array}{l}\text { F: TGTATACCCCTGGTGGGAGA } \\
\text { R: TCATAACTCCGGTCCCTCTG }\end{array}$ & 164 \\
\hline
\end{tabular}


Rønningen et al.

Supplemental Material

Supplementary Table 2. Primers used for ChIP-qPCR and genomic locations of amplicons (HG18 genome assembly)

\begin{tabular}{|c|c|c|c|c|}
\hline Gene & Area & Sequence & $\begin{array}{l}\text { Product } \\
\text { size }\end{array}$ & $\begin{array}{l}\text { Position (rel. } \\
\text { to TSS) }\end{array}$ \\
\hline \multirow[t]{3}{*}{ RARRES2 } & P1 & $\begin{array}{l}\text { F: } \\
\text { ATAGGTGTGGATGTGTGAAAGGA } \\
\text { R: CTGGGACATGTTACCTGGAGC }\end{array}$ & 70 & $-2288 /-2218$ \\
\hline & \begin{tabular}{|l|} 
P2 \\
\end{tabular} & $\begin{array}{l}\text { F: GGGGGCACCAGAAAGAAACT } \\
\text { R: CTCACAGCTGGGGAGTGAAG }\end{array}$ & 93 & $-1127 /-1034$ \\
\hline & TSS & $\begin{array}{l}\text { F: GCAAAGGCGTTCCTCATTCC } \\
\text { R: ACAGCGAGGCCAAGATTTCA }\end{array}$ & 139 & $+182 /+321$ \\
\hline \multirow[t]{3}{*}{ IL1B } & $E$ & $\begin{array}{l}\mathrm{F}: \text { CCACAGCACTTCCTTCCGAT } \\
\mathrm{R}: \text { TCCCTCCCTTAGTGCCAACT }\end{array}$ & 74 & $-3012 /-2938$ \\
\hline & $P$ & $\begin{array}{l}\text { F: GAGTGAGCCTCTGTGCAAGT } \\
\text { R: CTGCTTGGAGAGCAAGTCCA }\end{array}$ & 90 & $-1569 /-1479$ \\
\hline & TSS & $\begin{array}{l}\text { F: } \\
\text { AGCAGAAGTAGGAGGCTGAGA } \\
\text { R: TCCTTGCCCTTCCATGAACC }\end{array}$ & 149 & $-190 /-41$ \\
\hline \multirow[t]{2}{*}{ CCL2 } & $P$ & $\begin{array}{l}\text { F: CACTGCTGCCTGCTATGCTA } \\
\text { R: ACTAGTTGCCTGCTTGGGTC }\end{array}$ & 139 & $-846 /-985$ \\
\hline & TSS & $\begin{array}{l}\text { F: TCCTTTTCTGCAGTTTTCGCT } \\
\text { R: TGAGCAAATGGGGAGGATGG }\end{array}$ & 113 & $-200 /-313$ \\
\hline \multirow[t]{2}{*}{ CXCL1 } & $P$ & $\begin{array}{l}\text { F: TGCTTGGCTAGGAACACAGG } \\
\text { R: GGGCAGATGTCTCCACAACA }\end{array}$ & 72 & $-1316 /-1388$ \\
\hline & TSS & $\begin{array}{l}\text { F: GGCAGAAGGCGAATATCCCA } \\
\text { R: GTGTGGGGGTCCACTGATG }\end{array}$ & 87 & $-158 /-245$ \\
\hline \multirow[t]{2}{*}{ CXCL5 } & $P$ & $\begin{array}{l}\text { F: GTGACCAACGCTGTTTCCAC } \\
\text { R: } \\
\text { AGAGAGGGAGAGGAAGCTGG }\end{array}$ & 103 & $-1099 /-1202$ \\
\hline & TSS & $\begin{array}{l}\text { F: CGGAGATTGGAGGAGCGAAG } \\
\text { R: AAAAGGAGTGCAGAAGGCAC }\end{array}$ & 71 & $0 /+71$ \\
\hline \multirow[t]{2}{*}{ IL8 } & $P$ & $\begin{array}{l}\text { F: CCAGTGCACCACTTTCTGGA } \\
\text { R: CAAAGGCAGTTGGAGCAAGG }\end{array}$ & 150 & $-777 /-927$ \\
\hline & TSS & $\begin{array}{l}\text { F: ACCAAATTGTGGAGCTTCAGT } \\
\text { R: } \\
\text { TCAGGGCAAACCTGAGTCATC }\end{array}$ & 133 & $-107 /-240$ \\
\hline SF3A1 & $P$ & $\begin{array}{l}\text { F: CCCTACTCCCTTAGTCCCCC } \\
\text { R: GCAAGAAAAGGCAGGTTCCG }\end{array}$ & 108 & $-496 /-388$ \\
\hline
\end{tabular}

